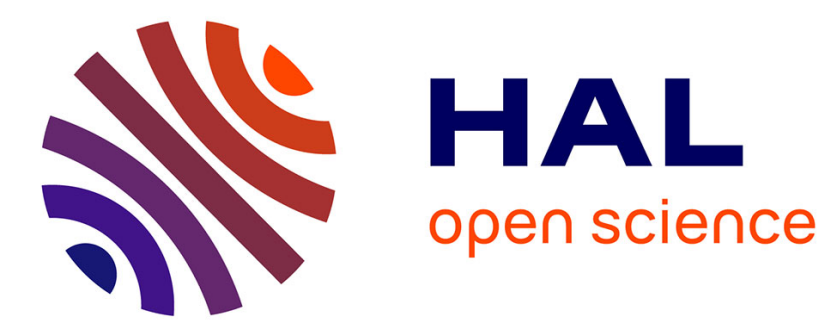

\title{
São Paulo, ou comment mettre à zéro l'affichage publicitaire
}

\author{
Nicolas Lechopier
}

\section{To cite this version:}

Nicolas Lechopier. São Paulo, ou comment mettre à zéro l'affichage publicitaire. Écologie \& politique : sciences, culture, société, 2010, 39, pp.87-100. hal-00576124

\section{HAL Id: hal-00576124 https://hal.science/hal-00576124}

Submitted on 12 Mar 2011

HAL is a multi-disciplinary open access archive for the deposit and dissemination of scientific research documents, whether they are published or not. The documents may come from teaching and research institutions in France or abroad, or from public or private research centers.
L'archive ouverte pluridisciplinaire HAL, est destinée au dépôt et à la diffusion de documents scientifiques de niveau recherche, publiés ou non, émanant des établissements d'enseignement et de recherche français ou étrangers, des laboratoires publics ou privés. 


\section{São Paulo, ou comment mettre à zéro l'affichage publicitaire}

\author{
NiCOLAS LECHOPIER
}

Au 19 siècle, São Paulo n'était encore qu'une bourgade. Elle connut l'opulence avec le commerce du café. Dans les années 1930, le café en surproduction fut brûlé dans les locomotives. Dans les années 1950, on fit fondre les voies de chemin de fer pour apporter de l'acier à l'industrie automobile. C'est aujourd'hui encore cette industrie-là qui porte la croissance économique de toute la région.

En un siècle, le nombre d'habitants de São Paulo a été multiplié par cent. Il y en a aujourd'hui plus de 20 millions dans la région métropolitaine, ce qui en fait la troisième agglomération du monde. São Paulo comme ville, comme lieu de vie de ses habitants, est le produit d'une modernisation brusque et non planifiée. Une ville bouillonnante au développement compulsif et cruel.

On raconte que lorsque les immigrants venus du Nordeste arrivent à São Paulo, trois choses frappent leur imagination : le flot ininterrompu de voitures et de camions; la hauteur des immeubles d'une ville « verticalisée»; et la profusion d'enseignes et de panneaux publicitaires géants, colorés, clignotants, tape-à-l'œil.

En septembre 2006, on estimait à environ cinq millions le nombre d'affiches (au sens large: enseignes, publicités, néons) visibles dans l'espace public. Outre les enseignes commerciales, on comptait environ 80000 panneaux publicitaires, sous forme de simple plaque, de bâche géante en façade ou de support métallique monté sur de hauts pylônes. La ville était littéralement recouverte d'affiches, son visage était celui de l'outdoor, le panneau de publicité extérieure.

Depuis avril 2007, quelques mois après le vote d'une loi municipale connue sous le nom de Cidade Limpa («ville propre»), São Paulo a radicalement changé de visage en se débarrassant de toute forme d'affichage publicitaire; et en réduisant en nombre et à une taille raisonnable les enseignes commerciales. Une certaine forme de pollution visuelle, mentale - a pris fin. Aujourd'hui, de nuit par temps clair, on peut de nouveau admirer les étoiles dans le ciel de la ville.

Un peu partout dans le monde, des tentatives similaires ont (eu) lieu, en tentant de limiter la quantité de publicité. Mais le cas de São Paulo est emblématique en raison de l'ampleur de la tâche accomplie avec
Nicolas Lechopier, enseignant, chercheur et militant antipublicitaire, a habité à São Paulo en 2008 et 2009. II remercie les Pr. Issao Minami et Takashi Fukushima de l'université de São Paulo, ainsi que Regina Monteiro, directrice de la section du paysage urbain (ENURB) à la mairie, pour leur bon accueil et le partage d'informations et d'archives. 
simplicité et radicalité. L'affichage publicitaire dans l'espace public a été littéralement remis à zéro à la façon d'un compteur chronométrique: il est probable qu'on le relâchera de nouveau et que l'affichage reviendra au compte-gouttes dans la ville. Mais pour le moment l'expérience mérite qu'on s'y arrête et peut-être qu'on s'en inspire.

Nettoyer la ville de toute publicité: quels sont les motifs qui justifient une telle mesure, quels sont les processus politiques qui y conduisent? Quelles en sont les conséquences économiques et écologiques? Quels effets cela produit, dans l'usage de la ville, dans l'expérience urbaine?

\section{Affichage publicitaire: un accroissement global}

Si la situation de São Paulo est évidemment singulière, l'envahissement par l'affichage publicitaire auquel a mis fin la loi Cidade Limpa est un phénomène plus général, dont on esquissera ici les principales caractéristiques. «Publicité» désigne le fait de rendre public quelque chose - un événement, une œuvre, une marchandise, un nom, un décret, etc. Bien avant que n'émergent les technologies de propagande perfectionnées au siècle dernier, la voix du crieur a été prolongée par des écrits et des images déployés sur la place publique.

L'affichage est en ce sens l'une des premières formes prise par la publicité - première chronologiquement, mais aussi en ordre d'importance. L'affiche placardée sur les murs reste l'outil le plus simple et le plus robuste pour rendre public quelque chose. $\mathrm{Ou}$, comme le disent les publicitaires, la «communication extérieure» ou «visuelle» est le moyen le plus efficace pour diffuser largement une campagne. L'affiche est relativement peu coûteuse à produire, elle peut être multipliée, déclinée à volonté. Elle ne présuppose pas que la «cible» achète un journal ou se pose devant un écran, seulement qu'elle se déplace dans l'espace où l'affiche émet en continu son message. Bien conçue, elle appelle le regard et génère un impact dans le cortex cérébral de celui ou celle qui la regarde (ne serait-ce que d'un œil distrait), impact qui augmente sa tendance à consommer un produit ou une marque.

En matière de publicité, la tendance de fond est à l'augmentation de la pression sur les individus. Cette intensification de la propagande reflète la nécessité pour le capitalisme d'accroître de manière constante la consommation. Les dispositifs publicitaires sont, en effet, non seulement la vitrine du système de production industriel mais surtout un maillon essentiel pour en écouler les produits : la publicité a pour fonc- 
tion macroéconomique principale de maintenir une propension élevée à consommer. Croissance économique et accroissement publicitaire s'engendrent l'un l'autre.

Cela se traduit par un envahissement des paysages par la pub. Comme la taille et la lisibilité typographique sont deux facteurs déterminants de l'attention à une affiche ${ }^{[1]}$, et comme chacune perd en efficacité à mesure qu'elle se partage l'attention des passants (le «temps de cerveau disponible») avec d'autres affiches ou dispositifs, cela génère une escalade: les publicitaires font croître en taille, en nombre et en visibilité les panneaux où les annonceurs diffusent leurs marques ou leurs produits. Le cercle est vicieux: on intensifie l'affichage pour compenser la diminution d'efficacité causée par la tendance globale à l'intensification. De cette hubris publicitaire, São Paulo recouverte de panneaux géants était devenue une preuve absurde.

\section{Le problème de la réglementation de l'affichage}

La réglementation de l'affichage est aussi un problème général. À l'échelon du parc naturel, de la grande ville ou du pays, il existe souvent des règles encadrant et limitant d'une façon ou d'une autre l'affichage dans l'espace public. Ces règles sont souvent insuffisantes, rarement respectées et ne parviennent pas à empêcher - faute peut-être de se donner précisément cet objectif - la colonisation de l'espace public (rues, trottoirs, murs, ciel) par la publicité.

Tracé à grands traits, ce processus de colonisation se déroule dans les villes de la façon suivante. Au départ, les affiches publicitaires se déploient dans l'espace public à l'initiative d'un secteur informel de petits entrepreneurs mettant à disposition de clients locaux des espaces sur lesquels afficher, conquis un par un. Dans un second temps, les afficheurs se regroupent et concentrent leur puissance publicitaire en établissant un réseau de panneaux et d'enseignes plus conséquent. Enfin, comme c'est le cas aujourd'hui dans de nombreuses métropoles, les afficheurs locaux laissent place à des multinationales de la communication extérieure (JCDecaux, Clear Channel, etc.), dont l'affichage est alors légalement inséré dans du «mobilier urbain» (arrêts de bus, horloges, etc.), ce qui produit des villes génériques ${ }^{[2]}$ habillées aux quatre coins du monde des mêmes couleurs, celles des grandes icônes commerciales. Un tel processus de privatisation et d'uniformisation de l'espace urbain reflète une option politique: rogner graduellement sur
[1] La taille est le facteur déterminant (suivi du contraste et de l'organisation de l'information) du niveau d'attention à la publicité, représentant $25 \%$ de la variance dans les scores de lecture selon C. Janiszewki, "The influence of display characteristics on visual exploratory search behavior ", Journal of Consumer Research, vol. 25, $\mathrm{n}^{\circ} 3,1998$, p. 290-301. [2] Même si les pratiques d'affichage publicitaire ne sont pas exactement uniformes à travers le monde, les paysages urbains à l'ère de la mondialisation ne sont pas aussi divers que les paysages naturels. On évoluera dans une architecture typographique dans des villes comme Shanghai, les forêts d'enseignes dans les villes dynamiques de l'Amérique latine, la présence pesante du mobilier urbain dans les villes françaises. Les affiches façonnent la condition urbaine, souvent avec l'assentiment des pouvoirs publics qui y voient une manière d'y mettre de l'ordre. 
[3] La loi 15.186/78 créa une commission de protection du paysage urbain (CPP) et la loi 15.364/78 définissait les règles suivant lesquelles les annonces publicitaires à l'air libre pouvaient être posées. [4] Source officielle, tribune de G. Kassab (actuel maire de São Paulo), Estado de São Paulo du 13 octobre 2006.

[5] Loi $13.525 \mathrm{du}$ 28 février 2003.

[6] II s'agissait d'autoriser les copropriétés d'immeubles à louer la façade pour le déploiement d'une bâche publicitaire pour financer des travaux de rénovation des façades. Ce genre de possibilités génère la plupart du temps des injustices (les habitants d'immeubles qui n'ont pas cette situation privilégiée doivent trouver une autre source de financement) et des abus (les bâches restent bien souvent après la fin des travaux). les espaces échappant aux activités de consommation et faire de tous les lieux des supports à marchandise.

À São Paulo, l'affichage publicitaire en était à la seconde phase, celui d'un ensemble bien organisé d'entreprises capables d'inonder l'espace public pour des campagnes assez massives (industrie automobile, boissons alcoolisées, grande distribution, etc.). L'affichage était encadré par une loi de protection du paysage urbain depuis 1978, votée alors qu'Olavo Setubal était maire de la ville. Toute pose d'un panneau était soumise à une autorisation préalable et à une inscription dans un registre municipal ${ }^{[3]}$. Évidemment, ce système n'a jamais fonctionné, faute de contrôleurs fiables et en nombre suffisant. Au début des années 2000, on comptait 80000 panneaux (dont $80 \%$ d'illégaux) ${ }^{[4]}$. En quelques décennies, São Paulo était devenue une ville littéralement invisible, aux façades méconnues de ses habitants. Un kaléidoscope publicitaire.

En 2003, lors du mandat de Marta Suplicy (PT, Parti des travailleurs), la loi de 1978 avait été refondue pour tenter de remédier à cette pollution visuelle ${ }^{[5]}$. Mais faute de remettre en cause l'existant, ce fut un échec cuisant. L'affichage a même sensiblement augmenté à partir de ce moment, puisque la loi, sans réussir à limiter l'affichage illégal, ouvrait en plus la possibilité légale de déployer des affiches géantes le long des façades en travaux ${ }^{[6]}$. Résultat: les immeubles les plus en vue de l'avenue Paulista (avenue principale de la ville, où l'on trouve les gratte-ciel les plus significatifs) en ont été entièrement recouverts.

Entre-temps, deux fronts s'étaient ouverts. D'abord dans le quartier d'Higienopolis, quartier juif ancien, avec un certain nombre d'immeubles à l'architecture remarquable mais peu valorisée car largement gâchée par l'affichage criard qui la recouvrait. L'avenue Angelica qui traverse ce quartier était bordée d'enseignes géantes et clignotantes, certaines au sommet de mâts inélégants plantés à même le trottoir («totems»). Des habitants revendiquant le droit à ne pas subir cette pollution publicitaire se sont organisés: ils ont occupé pendant 24 heures un chantier et ont confisqué les éléments du panneau avant qu'il ne soit monté. Des négociations furent conduites, sous l'égide du «promoteur de justice», entre les habitants et les entreprises et avec l'aide de la faculté d'architecture et d'urbanisme (université de São Paulo). Cela conduisit à supprimer les panneaux incriminés et à trouver des enseignes plus adéquates (plus petites, à échelle humaine).

Cet exemple de participation des habitants à la définition de leur paysage urbain a été commenté dans la presse comme un petit événement - l'esthétique du paysage urbain et le cadre de vie ne faisaient pas encore partie du champ d'action des politiques municipales - et fut lar- 
gement considéré comme une expérience concluante ${ }^{[7]}$. Les entreprises de l'avenue Angelica ayant accepté de réduire leurs enseignes à des dimensions raisonnables, mieux proportionnées à leur fonction d'indication d'un lieu commercial, ont vu qu'il en allait de leur intérêt bien compris et ont profité de cette opportunité pour associer leur image de marque à la valeur du respect de l'environnement urbain.

Le second front concernait les promoteurs immobiliers et apporta une leçon différente. Ceux-ci figuraient alors parmi les premiers responsables de la pollution visuelle en posant de grands panneaux temporaires sur les trottoirs et à tous les carrefours d'un quartier pour marquer le lieu de la grande vente anticipée d'appartements dans un immeuble en cours de construction. Certaines mairies d'arrondissement (subprefeituras) ont cherché au début des années 2000 à limiter ces panneaux temporaires en créant un autre système d'enregistrement et de contrôle. Mais l'expérience a montré que les contrôleurs étaient en trop faible nombre, souvent corrompus et de toute façon dans l'incapacité de mener à bien cette tâche. On en conclut que la seule option jouable était, non pas un encadrement - toujours complexe à mettre en ouvre - mais bien le principe d'une interdiction totale des panneaux ${ }^{[8]}$.

\section{La loi uville propre"}

En s'appuyant sur le double constat que le paysage urbain était une question pas si anodine aux yeux de la presse locale et des habitants, et que la voie de la légalisation d'une partie des dispositifs s'avérait un échec, un texte fut élaboré à partir de l'expérience des subprefeituras. Après d'âpres débats, l'assemblée municipale le votait en octobre 2006 à 45 voix contre une (celle du conseiller municipal issu du secteur publicitaire).

Ce texte a deux qualités indéniables: il est simple à comprendre dans ses principales dispositions et il est cohérent, donc crédible. La loi Cidade Limpa instaure en effet des règles aussi simples que radicales:

(1) Tous les dispositifs d'affichage publicitaire sont interdits - sauf s'ils sont placés sur du mobilier urbain (on y reviendra). Aujourd'hui, comme il n'y a pas (encore) de mobilier urbain dans la ville, déambuler dans les rues de São Paulo permet de faire cette rare expérience de ne jamais tomber dans une embuscade publicitaire;

(2) Les enseignes et pré-enseignes sont drastiquement limitées en nombre et en taille, selon la surface de l'entreprise concernée ${ }^{[9]}$.
[7] Voir le reportage «Beleza roubada", Revue du SESC São Paulo, n 114, novembre 2006, www. sescsp.org.br/sesc/revistas. [8] I. Minami, « Paisagem urbana de São Paulo. Publicidade externa e poluição visual », www. vitruvius.com.br/arquitextos/ arq000/esp074.asp.

[9] Pour une façade de moins de $10 \mathrm{~m}$, l'enseigne unique ne peut dépasser $1,5 \mathrm{~m}^{2}$. Pour une façade de 10 à $100 \mathrm{~m}$ elle est limitée à $4 \mathrm{~m}^{2}$. Et si la façade dépasse $100 \mathrm{~m}$, les différentes enseignes, séparées d'au moins $40 \mathrm{~m}$ les unes des autres, sont limitées à $10 \mathrm{~m}^{2}$. Pour d'autres détails, se reporter au site municipal: ww2.prefeitura.sp.gov.br// cidadelimpa. 
[10] Sepex, syndicat des entreprises de publicité extérieure de l'État de São Paulo.

[11] E. X. Pinto et N. Pucci Oliveira, "A importância do outdoor como meio de comunicação de massa e como mídia exterior ", communication faite à Intercom 2007. XXX Congresso Brasileiro de Ciências da Comunicação, Santos, www.intercom.org. br/papers/nacionais/2007/ resumos/R1572-1.pdf.
Cette loi est cohérente puisqu'elle s'applique à tout l'affichage dans l'espace public. À la mairie de São Paulo, on se souvient que l'une des plus fortes protestations a été celle des églises évangéliques. Sur le fronton des hangars où, de plus en plus souvent, elles invitent leurs fidèles à la ferveur religieuse, se déployaient de larges banderoles pieuses («Repentez-vous», etc.). Considérées comme de même nature que la propagande commerciale, la loi les a réduites à une taille humaine.

Lors des élections municipales d'octobre 2008, à l'issue desquelles le maire Gilberto Kassab (PSDB, Parti sociodémocrate centre droit) a été réélu contre la candidate du PT - ce qui n'est d'ailleurs peut-être pas tout à fait sans rapport avec le succès inattendu de cette politique de nettoyage de la publicité -, la question de la publicité électorale a fait l'objet d'un vif débat. La loi fédérale (définissant les modalités de campagne électorale) et la loi municipale (interdisant toute propagande dans l'espace public) entraient en conflit. Finalement, il fut établi que cette dernière primait et les partisans, autrefois colleurs d'affiche, furent forcés de se muer en porte-drapeaux agités au bord des rues et sur les ponts de la ville.

Seule exception dans la loi: les musées et les expositions culturelles, qui ont conservé le droit de déployer des banderoles, à condition que celles-ci prennent en compte l'intérêt du paysage urbain. La loi reste muette sur les graffitis et les murs peints par des artistes, ce qui permet aux pouvoirs locaux de fixer leur propre politique (encouragement ou répression). Une autre loi s'est chargée de constituer le délit de «tag» (autre pratique urbaine particulièrement visible à São Paulo) et d'en punir sévèrement les auteurs.

Les protestations les plus bruyantes face à cette loi venaient évidemment du secteur des afficheurs et, plus généralement, des entreprises de communication visuelle. Tous reconnaissaient des excès dans l'affichage publicitaire de São Paulo, mais leur position, représentée par un syndicat organisé ${ }^{[10]}$, était qu'on pouvait résoudre ce problème si l'on commençait par appliquer la loi existante. La façon dont ces entreprises se jouaient de la loi depuis des années ne laisse guère de doute sur leur sincérité, mais il semble en même temps qu'elles avaient pris conscience du problème de la saturation des espaces publics ${ }^{[11]}$. L'exemple de l'avenue Faria Lima, devenue dans les années 1990 le nouveau centre d'affaire de la ville, revient souvent comme l'un des symboles de ces excès dont personne ne pouvait tirer le moindre avantage: la multiplication et la juxtaposition des panneaux anéantissaient l'impact de chacun d'eux et l'on se retrouvait devant un ensemble totalement illisible. 
Évidemment, les afficheurs publicitaires étaient radicalement opposés à un texte bannissant tout affichage. Incrédules sur le fait que cette loi puisse être appliquée, ils n'ont pas réagi assez tôt. Des recours en inconstitutionnalité ont été déposés dans la foulée du vote, arguant du droit de propriété sur les panneaux et les enseignes, de la libre initiative des entrepreneurs privés du secteur de la communication visuelle et du principe de libre concurrence ${ }^{[12]}$. Mais la plus haute cour brésilienne conclut finalement que la loi municipale était raisonnable, proportionnée et n'instaurait pas de déséquilibre entre les intérêts publics et les intérêts privés. Ces multiples recours juridiques n'eurent pas d'autres effets que de repousser de trois mois le délai légal de mise en conformité. Dans les premiers mois de 2007, on fit démonter tous les panneaux, aux frais de leurs propriétaires.

\section{De la publicité comme pollution}

La loi «ville propre» considère la publicité comme l'une des sources majeures de pollution du paysage urbain (aux côtés des tags, des fils des réseaux téléphoniques et électriques pendants, des ordures apparentes, etc.). La notion de paysage urbain est définie comme l'espace aérien de la ville et la surface des éléments naturels et bâtis (eau, faune, flore, façades, signalisation urbaine, véhicules, publicités, enseignes, etc.) visibles par tout observateur situé dans les «aires d'usage communes $d u$ peuple ${ }^{[13]}$ ». Le paysage, c'est à la fois l'espace public et toutes les choses visibles qui s'y trouvent. Est «pollution visuelle» ce qui dégrade la qualité de ce paysage - en premier lieu l'affichage publicitaire excessif.

Mais on peut se demander: "pollution» est-il le mot juste pour désigner ce qui n'est peut-être, au pire, qu'une énorme faute de goût ${ }^{[14]}$ ? Rien de commun apparemment entre la pollution de l'eau ou la pollution atmosphérique (autres problèmes qu'affrontent au quotidien les Paulistas) et un paysage chargé d'affiches à la laideur duquel on sera plus ou moins sensible? L'affichage publicitaire et les enseignes n'ontils pas une vertu «décorative» en plus d'être «informatifs»?

Est «pollution» ce qui cause des dommages en dégradant le milieu de vie. Des études de cas ont montré que les affiches distraient les automobilistes et contribuent à causer des accidents de la circulation ${ }^{[15]}$. Qui plus est, les conditions esthétiques du cadre de vie affectent positivement ou négativement les activités sociales et économiques ${ }^{[16]}$. La pollution visuelle favorise le stress, la dépression, le sentiment d'étrangeté, elle affecte l'estime de soi en dégradant le sentiment du milieu ${ }^{[17]}$.
[12] La cour municipale de São Paulo (medida Cautelar contra a Lei 14.223, de setembro de 2006) donna en première instance raison à L\&C Outdoor Comunicação Visual Ltda et Publicidade Kilmes São Paulo Ltda, deux entreprises gérant des panneaux publicitaires animés. Cette décision fut cassée le 30 juillet 2008 par une juridiction supérieure, le tribunal de justice de São Paulo. Cette interprétation fut enfin confirmée au printemps 2009 par le tribunal suprême fédéral, la plus haute juridiction brésilienne. [13] Art. $2^{\circ}$ de la loi 14.223/2006.

[14] G. Cullen, Townscape, The Architectural Press, London, 1961, p. 153. [15] P. S. Leite Flores, Poluição visual e percepção da sinalização viaria, Thèse de doctorat, université de São Paulo, Faculdade de arquitectura e urbanismo, 2004.

[16] Les professionnels du tourisme savent que la pollution visuelle est un facteur déterminant de la satisfaction pour les visiteurs. Voir par exemple les commentaires sur le cas de Barcelone et de São Paulo dans M. Moyses et E. Wada, «São Paulo mais hospitaleira: projeto cidade limpa », TURyDES, vol. 1, $\mathrm{n}^{\circ} 1,2007$, www.eumed.net/ rev/turydes/01/index.htm. [17] Une enquête IBOPE conduite en 2006 concluait que quatre personnes sur cinq considèrent que la pollution visuelle est un problème «très grave» ou "grave». Voir A. Maurano, «A poluição visual e a nova lei paulista de publicidade ", Jus Navigandi, vol. 11, $\mathrm{n}^{\circ} 1324$, 15 fev. 2007, jus2.uol.com.br/ doutrina/texto.asp?id=9498. Voir aussi L. V. di Monte et F. M. Pacheco, Paisagem urbana de São Paulo e a poluição visual do século 
Enfin, l'envahissement publicitaire est assimilable à une pollution, dans la mesure où ceux qui la subissent ne sont ni informés ni consentants. Tout comme la fumée de tabac n'est une «pollution» que pour ceux qui la subissent sans avoir décidé d'être fumeurs, la publicité en est une puisqu'en l'occurrence on ne peut librement se soustraire aux messages qu'elle impose dans l'espace commun. La pollution, c'est un dommage environnemental subi. L'envahissement de l'espace public par l'affichage publicitaire est donc sans aucun doute une véritable pollution.

$X X I$, Scortecci, São Paulo, 2006 et C. Faccioni Mendes,

Paisagem urbana: uma mídia redescoberta, SENAC, São Paulo, 2006.

[18] Selon une enquête

(Datafolha) réalisée en août $2007,63 \%$ des sondés approuvaient la nouvelle loi, $54 \%$ estimaient qu'elle avait amélioré la ville tandis que pour $30 \%$ cela ne changeait rien et pour $14 \%$ la ville avait empiré.

[19] Dans la conclusion de leur intervention lors d'un congrès de sciences de la communication, E. X. Pinto et N. Pucci Oliveira (op. cit.) qualifient la récente loi de «totalement abusive et radicale dans son objectif de contrôler la communication de masse" et terminent par une splendide reductio ad hitlerum (seul Hitler serait capable de...).

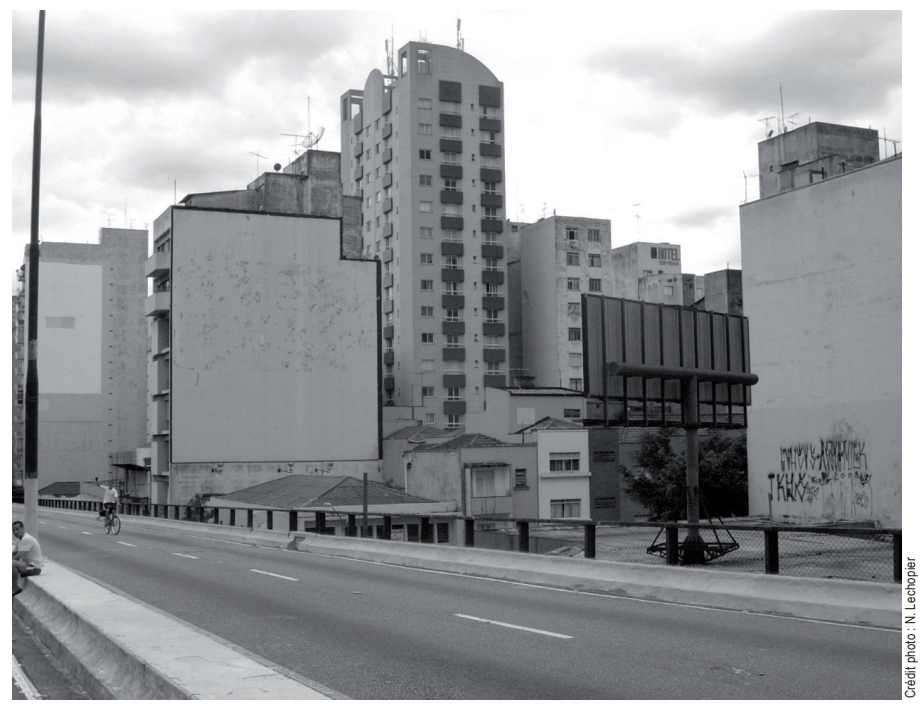

\section{Conséquences du nettoyage du paysage urbain}

Les discussions informelles avec les habitants de São Paulo aujourd'hui concordent avec un sondage ${ }^{[18]}$ qui montre que la suppression de la publicité de l'espace public a été et reste une mesure très populaire. Cependant, le débat public dans la presse et sur divers forums de discussion ne fut pas particulièrement serein. Mis à part les afficheurs eux-mêmes qui agitaient le risque d'une catastrophe économique et considéraient qu'il s'agissait là d'une mesure totalitaire que même les pires dictateurs de l'histoire n'avaient pas osé envisager ${ }^{[19]}$, les habitants semblent avoir eu du mal à concevoir dès le départ cette mesure comme quelque chose de positif. On s'était habitué à ce milieu urbain saturé de signes publicitaires, au point de les considérer comme indispensables à l'usage de la ville. Avec la suppression des panneaux 
et le remplacement des enseignes débordantes par de bien plus sobres et plus petites, on craignait notamment de ne plus s'y retrouver, de se perdre dans le dédale d'une ville sans lumières ni visage. On prédisait encore plus d'embouteillages à cause des hésitations des automobilistes. On agitait le spectre d'une chute de l'activité économique, faute d'orientation des consommateurs par l'affichage.

La loi est entrée en vigueur le premier janvier 2007. Nul ne savait si cette loi serait de celles qui «prennent» ou pas - au Brésil, il y a trop de ces lois généreuses et ambitieuses qui, pour une raison ou pour une autre, ne deviennent jamais effectives. Beaucoup avaient dans le doute maintenu leurs panneaux, au mieux sans y poser de nouvelles affiches. Lorsque les premières amendes tombèrent, ils durent s'en occuper précipitamment ${ }^{[20]}$. Des grues furent amenées en renfort pour l'occasion depuis les grandes villes des États voisins pour retirer des panneaux dont tout le monde semblait avoir oublié le degré d'encombrement qu'ils représentent. Le département de la voirie fut obligé de procéder à des coupures de circulation pour faciliter les travaux de démontage. Partout on commentait ce démantèlement inédit et la débauche de moyens qu'il impliquait. On trouve les traces de cette mutation soudaine dans les fameuses photos prises par T. de Marco ${ }^{[21]}$.

Étant donné l'ampleur de ce grand nettoyage urbain, la production de déchet a sans doute été gigantesque, mais cela n'a pas inquiété les services de l'urbanisme à la mairie. Cette loi fut une aubaine pour les carrossiers et les métalliers récupérant le métal des panneaux abandonnés. Les sacs à main confectionnés à partir des bâches de publicités abandonnées ont connu un grand succès jusqu'à New York. Mais en fait de recyclage, il y eut plutôt délocalisation: la loi ne s'appliquant qu'à la ville de São Paulo, les entreprises d'affichage ont pris les marchés des cités voisines, déplaçant leurs dispositifs les plus polyvalents (panneaux sur pylônes). Un nombre important de panneaux démontés à São Paulo a donc atterri dans les villes satellites jouxtant la capitale. Aujourd'hui, alors qu'aucune commune limitrophe n'a encore élaboré de règlement similaire, la présence de ces multiples panneaux géants d'un côté seulement de la route marque de façon frappante les frontières entre les différentes communes de la mégapole.

Sur le plan macroéconomique, il ne semble pas qu'il y ait eu d'impact visible sur le niveau de consommation des habitants, d'autant que les annonceurs se sont vraisemblablement reportés sur d'autres moyens de publicité (presse écrite, audiovisuelle, etc.). En revanche, la loi a clairement frappé un secteur essentiellement informel. Les afficheurs estimèrent à 350 millions d'euros le coût de la mesure pour les entreprises spécialisées ${ }^{[22]}$ et à environ 20000 le nombre de travailleurs touchés
[20] Une fois épuisé le délai de mise en conformité, des peines d'amendes de l'ordre de 4000 euros par panneau en infraction (montant doublant sur de courtes périodes de temps) ont été effectivement appliquées. [21] Voir l'album «São Paulo no logo " sur www.flickr.com/ photos/tonydemarco/sets. [22] Sergio Lopes (QG Propaganda), dans Estado de São Paulo, dimanche 8 avril 2007. 
[23] Aucun des produits dont ces panneaux faisaient la réclame (voitures, montres luxueuses, parfums, etc.) n'était évidemment accessible - sinon comme représentation imposée du bonheur conforme - aux personnes qui vivaient à leurs pieds. La richesse déployée par la publicité est d'abord la richesse fantasmée des autres. La publicité sert à générer une norme de comportement d'autant plus puissante que l'état idéal qu'elle désigne (être propriétaire d'une voiture sportive, d'une maison avec piscine, etc.) est éloigné de la situation de ceux qui la subissent. [24] Voir «Beleza roubada », op. cit.

[25] Backlight désigne les panneaux rétro-éclairés (par transparence), tandis que frontlight désigne ceux qui sont éclairés de l'extérieur par plusieurs lampes tournées vers l'affiche. [26] B. Begout, Zéropolis: l'expérience de Las Vegas, Allia, Paris, 2002. de près ou de loin par l'arrêt de l'activité d'affichage publicitaire dans l'espace public. D'après la mairie, certains d'entre eux auraient trouvé du travail dans les entreprises d'affichage des communes limitrophes, tandis que d'autres se seraient reconvertis, toujours dans le secteur informel. Triste répercussion cependant: les agences immobilières (celles-là mêmes qui contribuaient à la pollution urbaine lors de leurs ventes anticipées) paient aujourd'hui des jeunes gens pour se poster stratégiquement et agiter toute la journée une pancarte indiquant une vente en cours. Un panneau en mouvement, porté par un humain, n'est pas assimilé à un panneau publicitaire.

Avant que n'entre en vigueur la loi Cidade Limpa, l'une des favelas de la zone Sud de São Paulo était littéralement encerclée de ces panneaux géants tournés vers la route en contrebas ${ }^{[23]}$. Sauf à contourner cette barrière publicitaire, on ne pouvait même pas soupçonner l'existence d'un quartier habité à cet endroit. Dans ce quartier invisible où l'on ne peut aujourd'hui encore compter sur aucun aménagement urbain $^{[24]}$ - les habitants avaient tiré parti du halo des backlight et des frontlight ${ }^{[25]}$ qui les cachaient: panneaux et néons faisaient office d'éclairage public. Lorsqu'ils furent démantelés, le quartier perdit son éclairage public. Mais il retrouva une visibilité aux yeux des autres, il recommença d'exister dans le paysage urbain.

\section{Espace public et publicité}

De façon générale, et même si quelques petites affaires montées en épingle ont failli retourner l'opinion (par exemple, un avion de ligne peint aux couleurs d'un film d'animation américain fut interdit de survol de la ville, ce qui fut généralement présenté comme pointilleux et abusif), les gens estiment que la ville, depuis le retrait de la publicité, est plus agréable à vivre. Et dans une ville aux proportions monstrueuses comme São Paulo, ce n'est pas peu de chose. En vérité, en redécouvrant la ville une fois l'écran publicitaire tombé, on la trouva souvent laide, dégradée, négligée. Mais au moins - et c'est là une nouveauté - on en pensait quelque chose.

L'expérience du retrait des panneaux révélait ainsi que São Paulo n'est pas une zéropolis, comme le dit B. Begout à propos de Las Vegas ${ }^{[26]}$, une ville qui n'existe que comme reflet d'un fantasme projeté sur des bâches de plastiques. Cette ville invisible et depuis longtemps masquée retrouvait soudain un visage: un paysage certes peu enchanteur, mais véritable. 
À travers ce qui s'est passé à São Paulo, les panneaux publicitaires se révèlent dans leur fonction d'écran, dans le double sens de toile pour projeter l'image vendeuse et de masque de ce qui se trouve derrière. Ils tendent à remplacer la ville réelle par une ville fantasmée, intrinsèquement fausse, dans laquelle l'orientation est forcément erronée. La publicité dans l'espace public, loin d'être informative et fonctionnelle, désaligne l'espace urbain et en brouille la lisibilité. Elle disjoint l'espace visible (le paysage urbain perçu) de l'espace situé (où l'on est, où l'on va). Le géographe brésilien Milton Santos écrivait en ce sens que le paysage urbain devient sous l'effet de l'inflation publicitaire un «mensonge fonctionnel». Tous les lieux de travail, d'étude, de loisir, de la vie quotidienne sont conçus comme des marchandises. "La lecture du paysage, et a fortiori de l'espace, devient alors extrêmement difficile ${ }^{[27]} . »$

On retrouve là une caractéristique plus générale de la publicité qui, tant dans son contenu que dans ses supports, masque plus qu'elle ne montre. L'affichage publicitaire prétend être un outil de communication, mais en réalité il produit l'effet contraire. Comme le rappelle Hannah Arendt ${ }^{[28]}$, l'espace public n'est pas seulement l'espace physique délimité pour le mouvement des êtres humains. C'est aussi l'interposition des choses entre ceux qui cohabitent - choses qui leur donnent l'occasion de se rencontrer. Un peu comme la table à manger qui en même temps sépare et réunit les convives, l'espace public est fait d'intermédiaires. Quand c'est la publicité qui devient peu à peu l'intermédiaire principal de tout l'espace commun, ce dernier cesse d'être public. La publicité, en focalisant l'attention sur des «simulacres de présence ${ }^{[29]}$ », supprime les médiations au lieu de les susciter.

\section{Spéculations sur le mobilier urbain}

Au final, l'expérience de São Paulo est intéressante puisqu'elle fournit l'exemple concret d'une loi simple, effectivement appliquée et qui a des effets vertueux. Sur le plan des pratiques urbaines, elle représente un premier jalon pour la récupération de l'espace public en faisant naître un souci lié à la qualité du milieu de vie de millions de personnes ${ }^{[30]}$.

Cependant, il se pourrait que cette expérience soit de courte durée. La loi stipule en effet que toute publicité est interdite dans l'espace public - sauf sur le mobilier urbain. Le mobilier urbain c'est concrètement: (1) les horloges publiques, (2) les arrêts de bus et (3) un autre type de dispositif dont la nature n'a pas encore été définie. Pour l'instant, on
[27] M. Santos, Pensando o espaço do homen, Hucitech, São Paulo, 1997, p. 24.

[28] H. Arendt, Condition de I'homme moderne, Pocket, Paris, 1994.

[29] E. Landowski, Présences de l'autre, PUF, Paris, 1997. [30] Un terme imaginatif a été forgé pour signifier cet objectif: belezura. 
[31] Barcelone n'a pas réussi à éliminer la propagande publicitaire mais seulement à en changer la forme. Voir sur ce sujet J. Crosat, "La publicidad invade el espacio público de nuestras ciudades ", Rebelíon, 27 mars 2007, www.rebelion. org/noticia. php?id=48871.

[32] En 1972, JCDecaux introduisait un panneau de $2 \mathrm{~m}^{2}$ en forme de sucette, désormais nommé MUPI (mobilier urbain pour l'information, $120 \times 176 \mathrm{~cm}$ ). Le «MUPI Senior» est un panneau de $8 \mathrm{~m}^{2}$ surélevé au-dessus de la tête du passant et visible de loin, que l'on confond parfois avec le « $4 \times 3 \mathrm{~m}$ ».

[33] La partie vélos en libre-service n'était pas incluse dans la proposition. La question des transports à vélo dans la mégalopole brésilienne est assez délicate. Une autre expérimentation est en cours avec un parc d'une centaine de vélos financé par une entreprise d'assurances. ne peut voir dans la ville que quelques affiches publicitaires, de format 1 x $2 \mathrm{~m}$, surmontant de grosses horloges inélégantes disséminées aux grands carrefours urbains. En attendant que la justice se prononce sur un ancien litige, personne - hormis les tagueurs - ne touche aux panneaux apposés sur les rares endroits où l'arrêt de bus est marqué par la présence d'un abri. Quant à la troisième sorte de mobilier urbain, c'est probablement par là que reviendra l'affichage publicitaire à São Paulo.

La remise à zéro de la publicité dans le paysage urbain correspond en effet à un renouvellement profond du marché de l'affichage publicitaire. Des villes comme Barcelone ont connu ce processus d'une remise à plat de l'affichage (commencé en 1986 avec la campagne Barcelona, Posa't guapa et qui prit une quinzaine d'années) servant finalement à faire place nette pour un affichage publicitaire plus propre, policé et standardisé, celui des multinationales comme JCDecaux ${ }^{[31]}$, dont il est extrêmement difficile à une mairie de refuser l'offre: en échange du droit (éventuellement du monopole) d'affichage publicitaire, ce genre d'entreprise pose et entretient du mobilier urbain comme les abribus, paie à la mairie une redevance et peut installer en plus un système de vélos en libre-service. Suivant l'exemple d'une ville comme Lyon, le contrat est signé pour treize ans, ce qui assure à l'afficheur une juteuse rente de situation.

La notion même de «mobilier urbain » fait partie des facteurs expliquant comment ces multinationales de l'affichage ont réussi à s'implanter dans les moyennes et grandes métropoles un peu partout sur la planète. Cultivant l'ambiguïté entre le mobilier fonctionnel (les abribus, les toilettes publiques, etc.) et les purs supports d'affiches (les MUPI ${ }^{[32]}$ ), ces entreprises ont réussi durablement à coloniser l'espace public en se posant en champion de l'affichage chic et propre, au détriment de l'affichage irrégulier et brinquebalant des imprimeurs d'enseignes locaux. Pourtant, la pollution visuelle organisée, c'est encore de la pollution visuelle, une dégradation du paysage que subissent les personnes qui en sont la cible sans jamais l'avoir demandé et sans pouvoir s'en extraire.

Aujourd'hui, alors que les habitants de São Paulo apprécient unanimement de ne pas subir (en plus du reste) la laideur publicitaire, des enchères sont en cours. D’après les services municipaux concernés, la dernière proposition de JCDecaux pour São Paulo consistait à installer pas moins de 10000 MUPI en échanges de ces contreparties désormais classiques ${ }^{[33]}$. Mais cette proposition a été pour l'instant rejetée. Non seulement la voirie de São Paulo n'est pas faite pour les abribus standards de JCDecaux et encore moins pour des panneaux fixés au sol, mais surtout un retour de milliers de panneaux, même qualifiés de «mobilier urbain», donnerait le sentiment d'un net recul par rapport 
à la situation présente. À la mairie, on semble avoir pris conscience de l'importance politique du sujet. Alors que jusqu'à présent l'urbanisme de São Paulo était constamment considéré comme un parfait contre-exemple (transports publics misérables, gestion déplorable des ressources naturelles), la loi Cidade Limpa a suscité des éloges dans le monde entier ${ }^{[34]}$.

Une autre piste que celle du mobilier urbain est à l'étude. De plus en plus, la mairie de São Paulo invite les entreprises désireuses de s'afficher dans l'espace public à prendre en charge financièrement un aménagement urbain (daller une place publique, verdir un terre-plein, restaurer les abords d'un pont, etc.). En échange, ces entreprises gagnent le droit de poser une petite plaque indiquant leur contribution. Ce partenariat permet aux entreprises de soigner leur image de marque sans pour autant polluer visuellement l'espace public puisque là aussi l'apparence de ces dispositifs de propagande est drastiquement encadrée ${ }^{[35]}$.

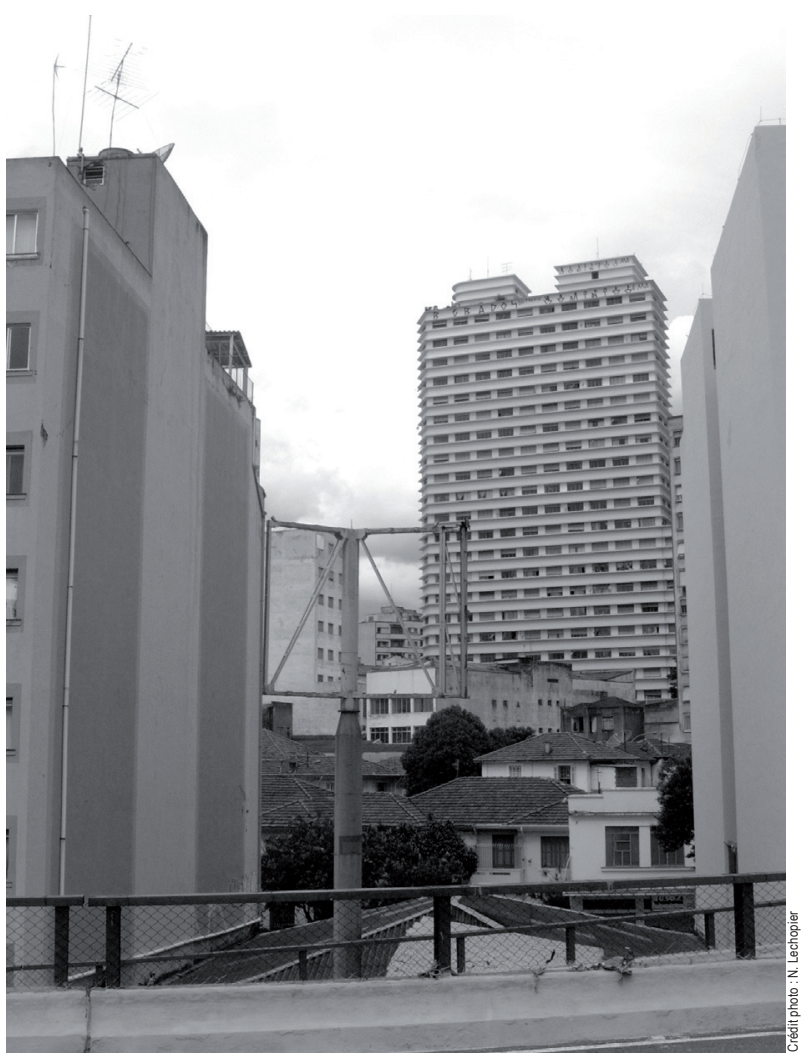

[34] Le maire de Bogota, l'une des villes phares de l'Amérique latine en matière d'aménagement urbain et de promotion des modes de transports alternatifs à la voiture, déclarait en 2008 que la loi Cidade Limpa était la politique urbaine la plus importante mise en place à São Paulo depuis dix ans. Cette initiative a été nominée pour un prix d'urbanisme. Et les responsables du paysage urbain de la mairie de São Paulo sont invités aux quatre coins du monde pour la présenter.

[35] Le décret $n^{\circ} 50.077 \mathrm{du}$ 6 octobre 2008 prévoit que ces plaques informatives ne devront pas dépasser $40 \mathrm{~cm}$ sur $60 \mathrm{~cm}$ et ne pas être situées à plus de $50 \mathrm{~cm}$ du sol. Ces plaques sont également limitées en nombre. Cette taille d'affichage, considérée comme suffisante et peu agressive, valide les propositions formulées par la plateforme en cours de constitution en France qui propose $50 \mathrm{~cm}$ sur $70 \mathrm{~cm}$ comme taille maximale de toute affiche dans l'espace public. Voir www.50x70.org. 
Ce genre d'«alternative» à l'affichage publicitaire n'est évidemment pas l'idéal. Faire financer des biens publics par des entreprises seulement désireuses de poser leur marque représente un risque en termes de pérennité (on peut d'ores et déjà voir à São Paulo des plates-bandes abandonnées surmontées d'une plaque en l'honneur du financeur). Et ce genre de partenariat contribue insidieusement à vider de son sens l'idée d'un espace public échappant à l'emprise du système marchand. L'expérience dira si, du point de vue de la qualité du paysage urbain, São Paulo a eu raison de prendre cette option. Face à la colonisation publicitaire, il nous faudrait avoir la ténacité d'un Bartleby ${ }^{[36]}$. Obstinément dire non, «j'aimerais mieux pas». 